\title{
Projetos de cidades inteligentes: $A$ rede de capacidade de construção da comunidade e o design thinking como viabilizadores para tornar o meio ambiente inteligente
}

\section{Vanessa Marie Salm}

Universidade do Estado de Santa Catarina. Av. Me. Benvenuta, 2007. Itacorubi. Florianópolis-SC, Brasil (CEP 88035-000). E-mail: vmsalm@hotmail.com.

Resumo. 0 meio ambiente das cidades vem demandando por projetos voltados para a adoção de estratégias que o torne inteligente. Com tal propósito, este artigo tem como objetivo criar uma proposta para tornar o meio ambiente inteligente por meio da rede de capacidade de construção da comunidade e do design thinking. Para tanto, realizou-se uma revisão de literatura sobre as temáticas relacionadas a esta proposta. Com base nisso, a proposta foi apresentada e discutida em três etapas, a saber: (i) levantamento e compreensão da realidade e das demandas/ problemas da comunidade; (ii) busca de soluções para demandas/problemas da comunidade, e (iii) criação do protótipo e entrega do plano de ação. Como principais resultados, a proposta viabilizará o enfoque interdisciplinar, a colaboração e participação de toda a comunidade na formulação de soluções para demandas ambientais fomentando, com isso, a conscientização ambiental, o desenvolvimento sustentável e o capital social na comunidade.

Palavra-chave: Meio ambiente inteligente; Rede; Comunidade.

\begin{abstract}
Smart city projects: The community capacity building network and design thinking as enablers for a smart environment. The environment of cities calls for projects aimed at adopting strategies that make them smart. With that purpose in mind, this article aims to create a proposal to make the environment smart through the network of community building capacity and design thinking (DT). Therefore, a literature review was carried out on the themes related to this proposal. From this, the proposal was presented and discussed in three stages, namely: (i) survey and understanding of the reality and demands/problems of the community; (ii) search for solutions to community demands/problems, and (iii) prototype creation and delivery of the action plan. As main results, the proposal Will enable na interdisciplinary approach, collaboration and participation of the entire community in the formulation of solutions for environmental demands, thus fostering
\end{abstract}

Recebido

$08 / 11 / 2021$

Aceito

$24 / 11 / 2021$

Disponível on line

$24 / 11 / 2021$

Publicado

$31 / 12 / 2021$

Acesso aberto

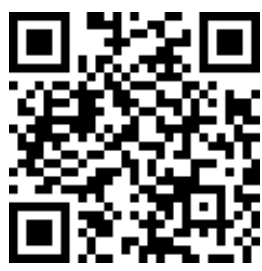

ORCID

(1) 0000-0003-1576-8541

Vanessa Marie Salm

ISSN 2359-1412/RBGAS-2021-0143/2021/8/20/7/1371

Rev. Bras. Gest. Amb. Sustent.

http://revista.ecogestaobrasil.net 
environmental awareness, sustainable development and social capital in the community.

Keywords: Smart environment; Network; Community.

\section{Introdução}

Os centros urbanos têm se transformado drasticamente desde a era industrial, o que vem acarretando, dentre outros fatores, no aumento populacional (Yigitcanlar, 2016). Os efeitos desse crescimento populacional têm gerado inúmeros desafios para o meio ambiente. Aletà et al. (2017) observam que os efeitos advindos do desenvolvimento urbano têm resultado na demanda por mais recursos naturais, no lançamento de gases poluentes e na geração de resíduos.

Diante dessas adversidades, Manville et al. (2014) destacam que as cidades inteligentes servem como uma estratégia para frear os impactos sociais e ambientais. Para os referidos autores, uma cidade inteligente está voltada para o bem-estar e para o desenvolvimento socioeconômico sustentável, fundamentando-se na construção e interação de aspectos como a tecnologia e o capital social e humano. No entanto, para que esses aspectos possam ser desenvolvidos, no cotidiano dos centros urbanos e de suas comunidades, é necessário que os cidadãos co-criem soluções, para suas demandas, através do restabelecimento do senso de pertencimento e do seu empoderamento (Oliveira e Campolargo, 2015).

Neste sentido, Schliwa (2019) observa que diversas têm sido as iniciativas que buscam revigorar a cidade inteligente, com enfoque mais recente para o olhar centrado no cidadão. No que tange essas iniciativas, a rede de capacidade de construção da comunidade e a aplicação da abordagem do design thinking podem servir de alicerces para projetos de cidades inteligentes, especificamente para a dimensão do meio ambiente inteligente.

No que se refere à rede de capacidade de construção da comunidade, Milward e Provan (2006) descrevem que o foco dessas redes é a criação do capital social para que a comunidade possa tratar das demandas atuais e futuras. Para os autores supracitados, essas redes possibilitam tornar a cidade mais resiliente e responsiva diante dos seus desafios.

Com relação ao design thinking, Brown e Wyatt (2010) definem como uma abordagem centrada no ser humano. Para tanto, o processo do design thinking, realizado de maneira não linear, é descrito por meio de três espaços: inspiração, ideação e implementação. Brown e Wyatt (2010), consideram que a inspiração tem relação com a motivação na busca por soluções para demandas ou oportunidades; já a ideação se associa à criação, desenvolvimento e testagem de ideias e, por fim, a implementação promove a adoção dessas ideias para o cotidiano dos indivíduos. Quando levada para a realidade das cidades inteligentes, Stimmel (2015) observa que o design thinking permite remodelar os desafios das cidades inteligentes, dando enfoque nas demandas dos cidadãos e em como a eficiência das cidades podem lhes ser úteis.

No que concerne aos projetos de cidades inteligentes, Fernandez-Anez et al. (2020), embasados nas melhores práticas e nos desafios das cidades, propõem que esses projetos possam ser estruturados em seis dimensões que abrangem, respectivamente, trinta e seis áreas e cento e dezoito ações dos projetos. Dentre as dimensões apresentadas, está a do meio ambiente inteligente. Para Manville et al. (2014), o meio ambiente inteligente engloba fatores que promovem a preservação e a conservação ambiental, dentre os quais se pode citar os recursos energéticos renováveis, a medição e fiscalização 
da poluição da água, do ar e sonora, as construções e gestão urbana verde, a promoção da reutilização e o correto descarte dos resíduos, entre outros.

Seguindo nesta linha, Fernandez-Anez et al. (2020) indicam que a dimensão do meio ambiente inteligente, em projetos de cidades inteligentes, deve compreender sete áreas que englobam diversas ações, conforme se apresenta na Tabela 1.

Tabela 1. Áreas e ações da dimensão do meio ambiente inteligente.

\begin{tabular}{|l|l|}
\hline Áreas & \multicolumn{1}{|c|}{ Ações } \\
\hline $\begin{array}{l}\text { Rede e monitoramento } \\
\text { ambiental }\end{array}$ & $\begin{array}{l}\text { Monitoramento ambiental, monitoramento de rede e aplicativos } \\
\text { para visualizar as informações coletadas. }\end{array}$ \\
\hline Eficiência energética & $\begin{array}{l}\text { Redes inteligentes, energia renovável, eficiência energética em } \\
\text { edifícios e distritos e eficiência energética em dispositivos públicos. }\end{array}$ \\
\hline $\begin{array}{l}\text { Planejamento urbano e e } \\
\text { reabilitação urbana }\end{array}$ & $\begin{array}{l}\text { Planejamento urbano em novos empreendimentos, remodelação } \\
\text { urbana, gestão urbana relacionada ao planejamento, participação } \\
\text { na gestão e planejamento urbano. }\end{array}$ \\
\hline $\begin{array}{l}\text { Edifícios inteligentes e e } \\
\text { renovação de edifícios }\end{array}$ & $\begin{array}{l}\text { Sustentabilidade em novas edificações e sustentabilidade na } \\
\text { reforma de edifícios. }\end{array}$ \\
\hline $\begin{array}{l}\text { Gestão de recursos } \\
\text { Proteção ambiental }\end{array}$ & $\begin{array}{l}\text { Gestão de resíduos, gerência de água, gestão de alimentos e } \\
\text { padrões de consumo. }\end{array}$ \\
\hline $\begin{array}{l}\text { Proteção dos recursos naturais, proteção de ecossistemas e } \\
\text { proteção da biodiversidade. } \\
\text { comportamental }\end{array}$ & $\begin{array}{l}\text { Ferramentas para mudança comportamental, ferramentas de } \\
\text { sensibilização e envolvimento em sustentabilidade medidas em } \\
\text { edifícios e espaços urbanos. }\end{array}$ \\
\hline
\end{tabular}

Fonte: Adaptado de Fernandez-Anez et al. (2020, p.84).

Conforme evidenciado na literatura acadêmica, verifica-se que a rede de capacidade de construção da comunidade e o design thinking podem contribuir para os projetos de cidades inteligentes. Entretanto, se faz necessário propor ações que contemplem as características dessa rede e os processos de design thinking voltados, especialmente, para o meio ambiente inteligente.

Diante disso, o objetivo deste artigo é criar uma proposta para tornar o meio ambiente inteligente por meio da rede de capacidade de construção da comunidade e do design thinking. Esta proposta tem na sua relevância oportunizar a articulação e o diálogo entre os membros da comunidade permitindo-lhes fomentar o capital social e co-criarem soluções para demandas do meio ambientes de suas cidades.

\section{Metodologia}

Realizou-se uma revisão de literatura, baseando-se principalmente nos autores referentes aos temas da rede de capacidade de construção da comunidade, do design thinking, e do meio ambiente inteligente.

Quanto à apresentação e discussão dos resultados, serão descritas as seguintes etapas: (i) levantamento e compreensão da realidade e das demandas/problemas da comunidade; (ii) busca de soluções para demandas/problemas da comunidade e (iii) criação do protótipo e entrega do plano de ação. Vale salientar que essas etapas, se embasam em Brown e Wyatt (2010), Milward e Provan (2006) e Fernandez-Anez et al. (2020). 
0 detalhamento das etapas supracitadas, conforme apresentado na figura 1, seguirá a seguinte ordem: correspondência ao processo do design thinking (inspiração, ideação e implementação), seus métodos e ferramentas, bem como as características da rede de capacidade de construção da comunidade que se inserem nessas etapas. Além disso, para cada uma das etapas, apresentar-se-á algumas das áreas e ações, da dimensão do meio ambiente inteligente, que elas podem abranger. Destaca-se, que serão apresentadas apenas as áreas e ações que englobam o escopo deste artigo. Na sequência, serão descritos os resultados esperados e discutidas as formas de aplicação e a contribuição para o meio ambiente inteligente. Por fim, vislumbra-se que esta proposta seja aplicada e desenvolvida no contexto de uma rede integrada por membros da comunidade e representantes de órgãos públicos, privados e sem fins lucrativos,

Figura 1. Conteúdo de cada uma das etapas da proposta.

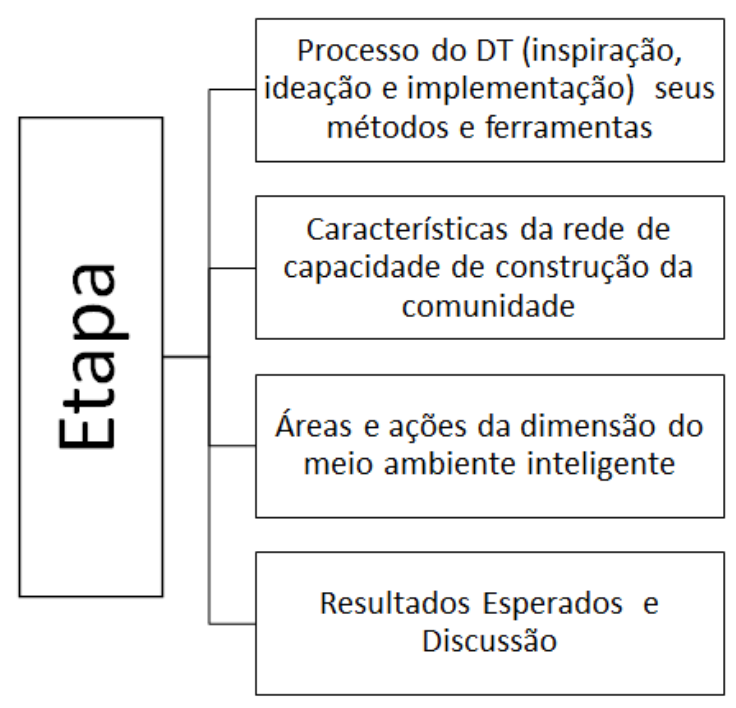

\section{Resultados e discussão}

A etapa de levantamento e compreensão da realidade e das demandas/problemas da comunidade tem como principal objetivo identificar e entender as principais demandas/problemas, relacionadas ao meio ambiente, enfrentados pela comunidade. No contexto do processo do design thinking, esta etapa faz referência à inspiração. Como sugestão de métodos aplicados, nesta conjuntura, a IDEO (2021), indica o "5 Por Quês", que permite realizar indagações sobre a razão de um problema/demanda em cinco ciclos, questionando-se sobre a razão apresentada anteriormente até completarse o quinto ciclo (Scartezini, 2009). Também, sugere-se a ferramenta "enquadramento" que"consiste em abordar o problema sob diferentes perspectivas e diversos ângulos, permitindo assim desconstruir crenças e suposições e quebrar seus padrões de pensamento, gerando novos insights" (Santanna et al., 2014, p. 24).

Com relação às características da rede de capacidade de construção da comunidade, esta etapa alinha-se com as necessidades atuais e futuras da comunidade, proporcionando atendê-las ao passo em que forem ocorrendo. A área do projeto contemplada nesta etapa é a de proteção ambiental, focando-se nas ações de proteção dos recursos naturais, dos ecossistemas e da biodiversidade. Esta etapa abrange a referida área e ações, pois elas são a motivação para o que se propõe nesta etapa. 
Como resultado esperado dessa etapa, pretende-se que se possa levantar e compreender uma demanda/problema, tendo como escopo as áreas e ações de projeto supracitadas e a situação atual e futura da comunidade. Esse escopo juntamente com a aplicação dos "5 Porquês" e o "enquadramento" adéquam com a natureza interdisciplinar do paradigma das cidades inteligentes, conforme observa Paskaleva (2018) ao relatar que esse paradigma, caracteriza-se pela aplicação de técnicas e métodos de diferentes áreas do conhecimento. Da mesma forma, os autores Micheli et al. (2019), descrevem a colaboração interdisciplinar como um dos atributos do DT.

Na segunda etapa desta proposta, busca de soluções para demanda/problema da comunidade, após entrarem em um consenso sobre uma demanda/problema relevante a ser resolvido, os membros da rede irão apresentar alternativas para a resolução da demanda/problema discutido na etapa anterior.

No que tange o processo de design thinking, esta etapa relaciona-se à ideação, pois ela foca em definir a demanda/oportunidade do projeto, elaborar o resumo do projeto e observar o grupo alvo no seu cotidiano (Tschimmel, 2012). Desta forma, sugere-se para facilitar essa etapa, o brainstorming, que permite a criação de novas ideias e vislumbrar novas possibilidades, e o mapeamento de jornada em que se analisa a vivência de uma determinada situação sob a perspectiva do cliente ou cidadão (Liedtka e Ogilvie, 2011).

Para tanto, além dos integrantes da rede já sugeridos no início desta seção, poderão estar envolvidos representantes de instituições de ensino e voluntários. Essa ampliação da rede está em consonância com uma das características da rede de capacidade de construção da comunidade, permite suprir demandas específicas que porventura possam surgir. No que tange à área do projeto, esta etapa adéqua-se à área de planejamento e reforma urbana, especificamente na remodelação urbana. Tendo em vista que, a rede buscará soluções para uma demanda/problema, no âmbito ambiental, da comunidade buscando restaurá-la de alguma maneira.

Quanto ao resultado esperado desta etapa, os membros da rede de diferentes áreas e com diferentes vivências e abordagens irão procurar formas para a resolução da demanda/problema que, de alguma forma, colaborará para a remodelação urbana.Este contexto é facilitado pelo mapeamento de jornada e brainstorming. Neste sentido, o resultado esperado desta etapa vai de encontro ao que Tschimmel (2012) sugere, que na ideação a equipe interdisciplinar socializa o que foi observado, e por meio de brainstorming busca apresentar soluções para a demanda/oportunidade complexa. Da mesma forma, o resultado esperado nesta etapa reverbera aquilo que observam Aletà et al. (2017) sobre o planejamento urbano na atualidade, foco da área de projeto nesta etapa, ser entendido como uma agregação em que ocorre a participação, a troca de ideias e a colaboração. Seguindo nesta linha, os resultados esperados para esta etapa também abrangem aquilo que Komninos (2014) salienta sobre as cidades inteligentes envolverem, dentre outros aspectos, as redes de colaboração nas comunidades que possibilitam a busca por solução das demandas e mecanismos eficazes dos insumos.

Na terceira etapa do projeto, criação do protótipo e entrega do plano de ação, primeiramente será selecionada, pelos agentes envolvidos na primeira etapa, uma das soluções e ideias apresentadas na etapa anterior. A partir disso, será criado um protótipo que deverá ser aprovado pelos agentes envolvidos. Com base no protótipo, serão feitos os devidos ajustes e, emseguida, serácriado um plano de ação para a futura execução do protótipo.

Ao que concerne o processo do design thinking, esta etapa se assemelha à da implementação. Para Tschimmel (2012), na implementação selecionam-se as melhores ideias para serem colocadas em um plano de ação, e por fim, buscam-se estratégias para comunicar e desenvolver a ideia. 
Tabela 2. Síntese das etapas.

\begin{tabular}{|c|c|c|c|c|}
\hline Etapa & $\begin{array}{l}\text { Processo de DT, } \\
\text { ferramentas e } \\
\text { métodos }\end{array}$ & $\begin{array}{l}\text { Características da } \\
\text { rede de } \\
\text { capacidade de } \\
\text { construção da } \\
\text { comunidade }\end{array}$ & $\begin{array}{c}\text { Áreas do } \\
\text { Projeto e ações } \\
\text { contempladas }\end{array}$ & $\begin{array}{c}\text { Contribuição } \\
\text { para o meio } \\
\text { ambiente } \\
\text { inteligente }\end{array}$ \\
\hline $\begin{array}{l}\text { Levantamento e } \\
\text { compreensão da } \\
\text { realidade e das } \\
\text { demandas/proble } \\
\text { mas da } \\
\text { comunidade }\end{array}$ & $\begin{array}{l}\text { Processo de DT: } \\
\text { Inspiração } \\
\text { Métodos/Ferrame } \\
\text { ntas: "5 Por Quês" } \\
\text { e "enquadramento" }\end{array}$ & $\begin{array}{l}\text { Atender as } \\
\text { necessidades atuais } \\
\text { e futuras da } \\
\text { comunidade, na } \\
\text { medida em que } \\
\text { forem ocorrendo }\end{array}$ & $\begin{array}{l}\text { Área: Proteção } \\
\text { ambiental } \\
\text { Ação: Proteção } \\
\text { dos recursos } \\
\text { naturais; de } \\
\text { ecossistemas e da } \\
\text { biodiversidade }\end{array}$ & $\begin{array}{l}\text { - Aplicação de } \\
\text { técnicas e métodos } \\
\text { de diferentes áreas } \\
\text { do conhecimento } \\
\text { (interdisciplinar) } \\
\text { para entendimento } \\
\text { da realidade das } \\
\text { demandas/problema } \\
\text { s da cidade e da } \\
\text { comunidade }\end{array}$ \\
\hline $\begin{array}{l}\text { Busca de soluções } \\
\text { para demandas/ } \\
\text { problemas da } \\
\text { ação na } \\
\text { comunidade }\end{array}$ & $\begin{array}{l}\text { Processo de DT: } \\
\text { Ideação } \\
\text { Métodos/ } \\
\text { Ferramentas: } \\
\text { Brainstorming e o } \\
\text { mapeamento de } \\
\text { jornada }\end{array}$ & $\begin{array}{l}\text { Ampliação da rede, } \\
\text { com diferentes } \\
\text { membros, para } \\
\text { suprir demandas } \\
\text { específicas que } \\
\text { possam surgir }\end{array}$ & $\begin{array}{l}\text { Área: } \\
\text { Planejamento } \\
\text { urbano e } \\
\text { reforma urbana } \\
\text { Ação: } \\
\text { Remodelação } \\
\text { urbana }\end{array}$ & $\begin{array}{l}\text { - Colaboração e } \\
\text { participação no } \\
\text { planejamento } \\
\text { urbano, } \\
\text { especificamente na } \\
\text { remodelação. } \\
\text { - Redes de } \\
\text { colaboração nas } \\
\text { comunidades e busca } \\
\text { conjunta por } \\
\text { soluções }\end{array}$ \\
\hline $\begin{array}{l}\text { Criação do } \\
\text { protótipo e } \\
\text { entrega do plano } \\
\text { de ação }\end{array}$ & $\begin{array}{l}\text { Processo de DT: } \\
\text { implementação } \\
\text { Métodos/ } \\
\text { Ferramenta: } \\
\text { protótipo em papel }\end{array}$ & $\begin{array}{l}\text { Constitui o capital } \\
\text { social na } \\
\text { comunidade }\end{array}$ & $\begin{array}{l}\text { Área: } \\
\text { Conscientização } \\
\text { e mudança } \\
\text { comportamental } \\
\text { Ação: } \\
\text { Ferramentas } \\
\text { para mudança } \\
\text { comportamental }\end{array}$ & $\begin{array}{l}\text { - Conscientização } \\
\text { ambiental } \\
\text { - Participação dos } \\
\text { indivíduos no } \\
\text { desenvolvimento } \\
\text { sustentável } \\
\text { - Fundamentar o } \\
\text { capital social }\end{array}$ \\
\hline
\end{tabular}

Quanto às ferramentas que podem facilitar esta etapa, sugere-se o protótipo em papel, por se enquadrar na realidade da rede de capacidade de construção da comunidade e por permitir tornar esta etapa mais colaborativa. Santanna et al. $(2014$, p. 26) definem essa ferramenta como "uma representação gráfica da solução, podem ser desenhos esquemáticos descrevendo o processo de negócio. Esse protótipo deve ser submetido à análise dos atores e, em um processo iterativo, ser melhorado".

Quanto à característica da rede de capacidade de construção da comunidade, esta etapa condiz com o intuito dessa rede que é constituir o capital social na comunidade. Indo ao encontro desta característica da rede, a área do projeto que será contemplada, é a da conscientização e mudança comportamental englobando a ação relacionada às ferramentas para mudança comportamental. 
Com relação ao resultado esperado dessa etapa, vislumbra-se que se fundamente o capital social na comunidade por meio de uma conscientização ambiental advinda das etapas anteriores e da implementação da solução proposta pela rede de capacidade de construção da comunidade. Neste sentido, nesta etapa, se prevê testar a solução através do protótipo em papel e, também, se apresentará um plano de ação para que essa implementação possa ser colocada em prática.

Desta forma, os resultados esperados nessa etapa alinham-se a algumas das características de cidades inteligentes propostas por Angelidou (2016), redes e o capital social e humano. Indo nesta linha, Kuzior e Sobotka (2019) indicam o capital social como forma de subsidiar a participação dos indivíduos na administração de uma cidade inteligente permitindo, assim, que haja o desenvolvimento sustentável na cidade.

Ao se finalizar a descrição das etapas que compreendem esta proposta, na Tabela 2 se apresentará uma síntese de cada uma das etapas com suas respectivas descrições norteadas sobre os pilares teóricos, embasados em Milward e Provan (2006), Brown e Wyatt (2010) e Fernandez-Anez et al. (2020), e contribuições para o meio ambiente inteligente.

\section{Conclusão}

0 presente artigo objetivou a criação de uma proposta para tornar o meio ambiente inteligente por meio da rede de capacidade de construção da comunidade e do design thinking. Tal proposta permitirá que se compreenda e solucione os desafios da comunidade relacionados ao meio ambiente através de uma perspectiva interdisciplinar, da co-criação, participação e colaboração dos membros da rede. Essa perspectiva permite à comunidade visualizar e antever desafios atuais e futuros, também, co-criar soluções para se remodelar a área urbana, embasando-se na ampliação da rede, na conscientização ambiental, na construção do capital social, empoderamento da comunidade e no desenvolvimento sustentável voltado para construção de um meio ambiente inteligente.

\section{Conflito de interesses}

A autora declara não haver conflito de interesses.

\section{Referências}

Aletà, N. B.; Alonso, C. M.; Ruiz, R. M. A. Smart mobility and smart environment in the Spanish cities. Transportation Research Procedia, v. 24, p. 163-170, 2017. https://doi.org/10.1016/j.trpro.2017.05.084

Angelidou, M. Four European smart city strategies. International Journal of Social Science Studies, v. 4, p. 18-30, 2016. https://doi.org/10.11114/ijsss.v4i4.1364

Brown, T.; Wyatt, J. Design thinking for social innovation. Stanford: Stanford Social Innovation Review, 2010.

Creswell, J. Projeto de pesquisa: métodos qualitativo, quantitativo e misto. Porto Alegre: Artmed, 2007.

Creswell, J. W.; Creswell, J. D. Projeto de pesquisa: métodos qualitativo, quantitativo e misto. São Paulo: Penso Editora, 2021. 
Fernandez-Anez, V.; Velazquez, G.; Perez-Prada, F.; Monzón, A. Smart city projects assessment matrix: Connecting challenges and actions in the Mediterranean Region. Journal of Urban Technology, v. 27, n. 4, p. 79-103, 2020. https://doi.org/10.1080/ 10630732.2018.1498706

IDEO. Methods. Design kit. 2021. Disponível em: <http://www.designkit.org/methods>. Acesso em: 08 out. 2021.

Komninos, N. The age of intelligent cities: Smart environments and innovation-for-all strategies. New York: Routledge, 2014.

Kuzior, A.; Sobotka, B. The role of social capital in the development of smart cities. Zeszyty Naukowe. Organizacja i Zarządzanie/Politechnika Śląska, 2019. https://doi.org/10.29119/1641-3466.2019.134.9

Liedtka, J.; Ogilvie, T. Designing for growth: A design thinking tool kit for managers. New York: Columbia University Press, 2011.

Manville, C.; Cochrane, G.; Cave, J.; Millard, J.; Pederson, J. K.; Thaarup, R. K.; Kotterink, B. Mapping Smart Cities in the EU. Bruxelas: European Parliament, 2014. Disponível em: <https://www.europarl.europa.eu/RegData/etudes/etudes/join/2014/507480/IPOLITRE_ET(2014)507480_EN.pdf>. Acesso em: 08 out. 2021.

Micheli, P.; Wilner, S. J.; Bhatti, S. H.; Mura, M.; Beverland, M. B. Doing design thinking: Conceptual review, synthesis, and research agenda. Journal of Product Innovation Management, v. 36, n. 2, p. 124-148, 2019. https://doi.org/10.1111/jpim.12466

Milward, H. B.; Provan, K. G. A manager's guide to choosing and using collaborative networks. Washington, DC: IBM Center for the Business of Government, 2006.

Oliveira, Á.; Campolargo, M. From smart cities to human smart cities. 48th Hawaii International Conference on System Sciences, IEEE, p. 2336-2344, 2015. https://doi.org/10.1109/HICSS.2015.281

Paskaleva, K. E-governance as an enabler of the smart city. In: Deakin, M. (Ed.). Smart cities: Governing and modelling the transition. New York: Routledge, 2018. p. 45-63.

Santanna, I. C. B. S.; Santanna Filho, J. F.; Pimenta, R. B.; Soriano-Sierra, E. Design thinking como ferramenta para melhoria em processos de negócios. Espacios, v. 35, n. 6, p. 19, 2014.

Scartezini, L. M. B. Análise e melhoria de processos. Goiânia: O Autor, 2009.

Schliwa, G. Smart cities by design? Interrogating Design thinking for citizen participation. In: Cardullo, P.; Di Feliciantonio, C.; Kitchin, R. (Eds.). The right the smart city. Bingley: Emerald, 2019. https://doi.org/10.1108/978-1-78769-139-120191011

Stimmel, C. Building smart cities. Boca Raton: Auerbach Publications, 2015.

Tschimmel, K. Design thinking as an effective toolkit for innovation. ISPIM Conference Proceedings, The International Society for Professional Innovation Management (ISPIM), 2012. 
Vergara, S. C. Tipos de pesquisa em administração. Rio de Janeiro: EBAP/FGV, 1990. (Caderno 52).

Yigitcanlar, T. Technology and the city: Systems, applications and implications. London: Routledge, 2016. 